\title{
Localized gravity and higher curvature terms
}

\author{
Olindo Corradini, Zurab Kakushadze \\ C.N. Yang Institute for Theoretical Physics, State University of New York, Stony Brook, NY 11794, USA \\ Received 6 September 2000; accepted 9 October 2000 \\ Editor: M. Cvetič
}

\begin{abstract}
We consider localization of gravity in smooth domain wall solutions of gravity coupled to a scalar field with a generic potential in the presence of the Gauss-Bonnet term. We discuss conditions on the scalar potential such that domain wall solutions are non-singular. We point out that the presence of the Gauss-Bonnet term does not allow flat solutions with localized gravity that violate the weak energy condition. We also point out that in the presence of the Gauss-Bonnet term infinite tension flat domain walls violate positivity. In fact, for flat solutions unitarity requires that on the solution the scalar potential be bounded below. (C) 2000 Elsevier Science B.V. All rights reserved.
\end{abstract}

\section{Introduction}

In the Brane World scenario the Standard Model gauge and matter fields are assumed to be localized on branes (or an intersection thereof), while gravity lives in a larger dimensional bulk of space-time [1-12]. The volume of dimensions transverse to the branes is automatically finite if these dimensions are compact. On the other hand, the volume of the transverse dimensions can be finite even if the latter are non-compact. In particular, this can be achieved by using [13] warped compactifications [14] which localize gravity on the brane. A concrete realization of this idea was given in [15].

One motivation for considering such unconventional compactifications is the moduli problem. In particular, the extra dimensions in such scenarios are non-compact while their volume is finite and fixed in terms of other parameters in the theory such as those in the scalar potential. That is, the expectation values of the scalars descending from the components of the higher dimensional metric corresponding to the extra dimensions are actually fixed.

Recently in [16] one of us considered localization of gravity arising in ( $D-1)$-dimensional (smooth) domain wall solutions in the system of $D$-dimensional Einstein-Hilbert gravity coupled to a single real scalar field with a generic scalar potential. In particular, [16] discussed conditions on the scalar potential such that the corresponding domain wall solutions are non-singular (in the sense that singularities do not arise at finite values of the coordinate transverse to the domain wall). The usual kink type of solutions are non-singular as they interpolate between two adjacent local AdS minima of the scalar potential. On the other hand, there exist other non-singular solutions

E-mail addresses: olindo@insti.physics.sunysb.edu (O. Corradini), zurab@insti.physics.sunysb.edu (Z. Kakushadze). 
(subject to the aforementioned non-singularity conditions on the scalar potential) which do not interpolate between AdS minima. In fact, such solutions exist even for potentials which have no minima at all and are unbounded below. Domain walls of this type have infinite tension.

In this paper we study effects of higher curvature bulk terms on the domain wall solutions of the aforementioned types. As was pointed out in [16], adding arbitrary higher curvature combinations would generically lead to delocalization of gravity. Moreover, if we truncate the bulk action at any finite higher derivative level, then generic higher curvature terms would lead to the appearance of ghosts in the Hilbert space. To avoid these difficulties, one might consider adding special "topological" combinations which do not spoil unitarity [17,18]. In this paper we focus on the simplest non-trivial term of this type, namely, the Gauss-Bonnet combination. ${ }^{1}$ Even though it is a total derivative in four dimensions (in particular, it is the four-dimensional Euler invariant), it is non-trivial in higher dimensions. Nonetheless, when expanded around a flat Minkowski metric, the Gauss-Bonnet term does not give rise to corrections to the propagator, so that ghost are not introduced. Another feature of the Gauss-Bonnet combination is that it can be supersymmetrized.

Thus, even though it is a special combination of higher curvature terms, studying domain walls with localized gravity in the presence of the Gauss-Bonnet term gives some insight into the higher curvature effects on such domain walls. Thus, for instance, we point out that the presence of the Gauss-Bonnet term does not allow flat solutions with localized gravity that violate the weak energy condition (that is, the analog of the $c$-theorem [23]). We also point out that in the presence of the Gauss-Bonnet term infinite tension flat domain walls violate positivity. In fact, for flat solutions unitarity requires that (on the solution) the scalar potential be bounded below.

The rest of this Letter, which is essentially a generalization of [16], is organized as follows. In Section 2 we describe the setup within which we will discuss solutions with localized gravity. In Section 3 we discuss solutions with vanishing $(D-1)$-dimensional cosmological constant. Section 4 contains concluding remarks.

\section{Setup}

In this section we discuss the setup within which we will discuss solutions with localized gravity. Thus, consider a single real scalar field $\phi$ coupled to gravity with the following action: ${ }^{2}$

$$
S=M_{P}^{D-2} \int d^{D} x \sqrt{-G}\left[R+\lambda\left(R^{2}-4 R^{M N} R_{M N}+R^{M N R S} R_{M N R S}\right)-\frac{4}{D-2}(\nabla \phi)^{2}-V(\phi)\right],
$$

where $M_{P}$ is the $D$-dimensional (reduced) Planck mass, and the term multiplied by a free parameter $\lambda$ is the Gauss-Bonnet combination. ${ }^{3}$ The equations of motion read:

$$
\begin{aligned}
& \frac{8}{D-2} \nabla^{2} \phi=V_{\phi}, \\
& R_{M N}-\frac{1}{2} G_{M N} R-\frac{1}{2} \lambda G_{M N}\left(R^{2}-4 R^{M N} R_{M N}+R^{M N R S} R_{M N R S}\right) \\
& \quad+2 \lambda\left(R R_{M N}-2 R_{M S} R_{N}^{S}+R_{M R S T} R_{N}^{R S T}-2 R^{R S} R_{M R N S}\right)
\end{aligned}
$$

\footnotetext{
1 Discontinuous domain walls (that is, domain walls with $\delta$-function-like brane sources with non-zero tension which explicitly break diffeomorphism invariance) in the presence of bulk Gauss-Bonnet term were discussed in [19-21]. In this Letter we will not discuss such domain walls (and, therefore, we will not have to deal with the issues recently pointed out in [22]). Rather, we will focus on smooth domain walls (without any $\delta$-function brane sources) which break diffeomorphisms spontaneously.

2 Here we focus on the case with one scalar field for the sake of simplicity. In particular, in this case we can absorb a (non-singular) metric $Z(\phi)$ in the $(\nabla \phi)^{2}$ term by a non-linear field redefinition. This cannot generically be done in the case of multiple scalar fields $\phi^{i}$, where one must therefore also consider the metric $Z_{i j}(\phi)$.

3 We are using the conventions $R_{N R S}^{M}=\Gamma_{N R, S}^{M}-\Gamma_{N S, R}^{M}+\Gamma_{R T}^{M} \Gamma_{N S}^{T}-\Gamma_{S T}^{M} \Gamma_{N R}^{T}$, and $R_{M N}=R_{M P N}^{P}$.
} 


$$
=\frac{4}{D-2}\left[\nabla_{M} \phi \nabla_{N} \phi-\frac{1}{2} G_{M N}(\nabla \phi)^{2}\right]-\frac{1}{2} G_{M N} V .
$$

The subscript $\phi$ in $V_{\phi}$ denotes derivative w.r.t. $\phi$.

In the following we will be interested in solutions to the above equations of motion with the warped [14] metric of the following form:

$$
d s^{2}=\exp (2 A) d \tilde{s}^{2}+d y^{2},
$$

where $y \equiv x^{D}$, the warp factor $A$, which is a function of $y$, is independent of the coordinates $x^{\mu}, \mu=1, \ldots, D-1$, and the $(D-1)$-dimensional interval is given by

$$
d \tilde{s}^{2}=\tilde{g}_{\mu \nu} d x^{\mu} d x^{\nu},
$$

with the $(D-1)$-dimensional metric $\tilde{g}_{\mu \nu}$ independent of $y$.

With the above ansatz, we have:

$$
\begin{aligned}
& R_{\mu \nu}=\widetilde{R}_{\mu \nu}-\exp (2 A)\left[A^{\prime \prime}+(D-1)\left(A^{\prime}\right)^{2}\right] \tilde{g}_{\mu \nu}, \\
& R_{D D}=-(D-1)\left[A^{\prime \prime}+\left(A^{\prime}\right)^{2}\right], \\
& R_{\mu D}=0, \\
& R_{v \rho \sigma}^{\mu}=\widetilde{R}_{v \rho \sigma}^{\mu}+\left(A^{\prime}\right)^{2} \exp (2 A)\left[\delta_{\sigma}^{\mu} \tilde{g}_{\rho \nu}-\delta_{\rho}^{\mu} \tilde{g}_{\nu \sigma}\right], \\
& R_{v \rho \sigma}^{D}=0, \\
& R_{\nu D \sigma}^{D}=-\exp (2 A)\left[A^{\prime \prime}+\left(A^{\prime}\right)^{2}\right] \tilde{g}_{\nu \sigma},
\end{aligned}
$$

where prime denotes derivative w.r.t. $y$. Also, the $(D-1)$-dimensional (“tilded") quantities such as $\widetilde{R}_{\mu \nu}$ and $\widetilde{R}_{\mu \nu \sigma \tau}$ are calculated w.r.t. the $(D-1)$-dimensional metric $\tilde{g}_{\mu \nu}$.

In the following we will be interested in solutions where $\phi$ depends non-trivially on $y$. From the above equations it then follows that $\phi$ is independent of $x^{\mu}$. The equations of motion for $\phi$ and $A$ then become:

$$
\begin{aligned}
& \frac{8}{D-2}\left[\phi^{\prime \prime}+(D-1) A^{\prime} \phi^{\prime}\right]-V_{\phi}=0, \\
& (D-1)(D-2)\left(A^{\prime}\right)^{2}\left[1-(D-3)(D-4) \lambda\left(A^{\prime}\right)^{2}\right]-\frac{4}{D-2}\left(\phi^{\prime}\right)^{2}+V \\
& \quad-\frac{D-1}{D-3} \tilde{\Lambda} \exp (-2 A)\left[1-2(D-3)(D-4) \lambda\left(A^{\prime}\right)^{2}\right]-\lambda \tilde{\chi} \exp (-4 A)=0, \\
& (D-2) A^{\prime \prime}\left[1-2(D-3)(D-4) \lambda\left(A^{\prime}\right)^{2}+2 \frac{D-4}{D-2} \lambda \tilde{\Lambda} \exp (-2 A)\right]+\frac{4}{D-2}\left(\phi^{\prime}\right)^{2} \\
& \quad+\frac{1}{D-3} \tilde{\Lambda} \exp (-2 A)\left[1-2(D-3)(D-4) \lambda\left(A^{\prime}\right)^{2}\right]+\frac{2 \lambda}{D-1} \tilde{\chi} \exp (-4 A)=0 .
\end{aligned}
$$

The first equation is the dilaton equation of motion, the second equation is the $(D D)$ component of (3), and the third equation is a linear combination of the latter and the $(\mu \nu)$ component of (3). In fact, the $(\mu \nu)$ component of (3) implies that $\tilde{\Lambda}$ is a constant, and is nothing but the cosmological constant of the $(D-1)$-dimensional manifold, which is therefore an Einstein manifold, described by the metric $\tilde{g}_{\mu \nu}$. Our normalization of $\tilde{\Lambda}$ is such that the $(D-1)$-dimensional metric $\tilde{g}_{\mu \nu}$ satisfies Einstein's equations

$$
\widetilde{R}_{\mu \nu}-\frac{1}{2} \tilde{g}_{\mu \nu} \widetilde{R}=-\frac{1}{2} \tilde{g}_{\mu \nu} \tilde{\Lambda}
$$

Moreover, the quantity

$$
\tilde{\chi} \equiv \widetilde{R}^{2}-4 \widetilde{R}_{\mu \nu}^{2}+\widetilde{R}_{\mu \nu \sigma \tau}^{2}
$$


is also a constant (for $\lambda \neq 0) .{ }^{4}$ Note that for $D-1=4$ the quantity $\tilde{\chi}$ is the Euler invariant for the $(D-1)$ dimensional manifold described by the metric $\tilde{g}_{\mu \nu}$. Finally, the aforementioned $(D-1)$-dimensional Einstein manifold must be such that

$$
\widetilde{R}_{\mu \rho \sigma \tau} \widetilde{R}_{\nu}^{\rho \sigma \tau}=\frac{1}{D-1} \widetilde{R}_{\alpha \beta \rho \sigma}^{2} \tilde{g}_{\mu \nu}
$$

This condition is automatically satisfied for maximally symmetric Einstein manifolds.

Note that we have only two fields $\phi$ and $A$, yet we have three Eqs. (12), (13) and (14). However, only two of these equations are independent. This can be seen as follows. Using the second equation one can express $\phi^{\prime}\left(A^{\prime}\right)$ via $A^{\prime}\left(\phi^{\prime}\right)$ and $V$. One can then compute $\phi^{\prime \prime}\left(A^{\prime \prime}\right)$ and plug it in the first (third) equation. This equation can then be seen to be automatically satisfied as long as the third (first) equation is satisfied. As usual, this is a consequence of Bianchi identities.

\section{Solutions with $(D-1)$-dimensional Poincaré invariance}

In this section we discuss solutions of the aforementioned equations with $\tilde{\Lambda}=0$ and $\tilde{\chi}=0$. In this case the equations of motion read:

$$
\begin{aligned}
& (D-1)(D-2)\left(A^{\prime}\right)^{2}\left[1-(D-3)(D-4) \lambda\left(A^{\prime}\right)^{2}\right]-\frac{4}{D-2}\left(\phi^{\prime}\right)^{2}+V=0, \\
& (D-2) A^{\prime \prime}\left[1-2(D-3)(D-4) \lambda\left(A^{\prime}\right)^{2}\right]+\frac{4}{D-2}\left(\phi^{\prime}\right)^{2}=0 .
\end{aligned}
$$

As in the $\lambda=0$ case, we can rewrite these equations in terms of the following first order equations

$$
\begin{aligned}
& \phi^{\prime}=\alpha W_{\phi}\left(1-\lambda \kappa W^{2}\right), \\
& A^{\prime}=\beta W,
\end{aligned}
$$

where

$$
\begin{aligned}
& \alpha \equiv \epsilon \frac{\sqrt{D-2}}{2}, \\
& \beta \equiv-\epsilon \frac{2}{(D-2)^{3 / 2}}, \\
& \kappa \equiv 2(D-2)(D-3) \beta^{2},
\end{aligned}
$$

and $\epsilon= \pm 1$. Moreover, the scalar potential $V$ is related to the function $W=W(\phi)$ via

$$
V=\left[W_{\phi}^{2}+\eta\right]\left(1-\lambda \kappa W^{2}\right)^{2}-\eta,
$$

where

$$
\eta \equiv \frac{(D-1)(D-2)}{4 \lambda(D-3)(D-4)}
$$

Note that for $\lambda>0$ the potential (25) is bounded below [19]. Also note that in the $\lambda \rightarrow 0$ limit from (25) we recover the familiar expression $V=W_{\phi}^{2}-\gamma^{2} W^{2}$, where $\gamma^{2} \equiv 4(D-1) /(D-2)^{2}$.

\footnotetext{
4 If the corresponding Einstein manifold is maximally symmetric, then we have $\widetilde{R}_{\mu \nu \rho \sigma}=\tilde{\Lambda}\left(\tilde{g}_{\mu \rho} \tilde{g}_{\nu \sigma}-\tilde{g}_{\mu \sigma} \tilde{g}_{\nu \rho}\right) /(D-2)(D-3)$, and $\tilde{\chi}=(D-1)(D-4) \tilde{\Lambda}^{2} /(D-2)(D-3)$. Generally, however, this Einstein manifold need not be maximally symmetric.
} 
Note that (19) implies the following condition:

$$
A^{\prime \prime}\left[1-2(D-3)(D-4) \lambda\left(A^{\prime}\right)^{2}\right] \leqslant 0 .
$$

It then follows that, since $A$ as well as its derivatives are continuous, $A^{\prime \prime}$ cannot change sign. That is, we have the following possibilities. If $\lambda$ is negative, then we necessarily have $A^{\prime \prime} \leqslant 0$. If $\lambda$ is positive, then we can have $A^{\prime \prime} \leqslant 0$ subject to the following additional requirement: $\left(A^{\prime}\right)^{2} \leqslant \beta^{2} / \lambda \kappa$. In this case gravity is localized as long as $A$ goes to $-\infty$ at $y \rightarrow \pm \infty$ fast enough. Another possibility (for $\lambda>0$ ) is that $A^{\prime \prime} \geqslant 0$, and $\left(A^{\prime}\right)^{2} \geqslant \beta^{2} / \lambda \kappa$. Note that in this case gravity is not localized (as $A$ goes to $+\infty$ at $y \rightarrow \pm \infty$ ). Thus, the presence of the Gauss-Bonnet term does not allow flat solutions with localized gravity that violate the weak energy condition (that is, the analog of the $c$-theorem [23]).

\subsection{Non-singularity conditions}

In this subsection we would like to discuss the conditions on $W$ such that the corresponding solutions do not blow up at finite values of $y$. More precisely, in this section we will focus on solutions such that $\phi$ is non-singular ${ }^{5}$ at finite $y$. To begin with note that if $V$ is non-singular, which we will assume in the following, then $W$ and $W_{\phi}$ should (generically) be non-singular as well. This then guarantees that solutions are continuous for finite values of $\phi$. However, a priori it is still possible that $\phi$ blows up at finite values of $y$.

The equation we would like to study here is

$$
\phi^{\prime}=\alpha Y_{\phi},
$$

where

$$
Y \equiv W-\frac{\lambda \kappa}{3} W^{3}
$$

In the following we will be interested in the cases where gravity is localized. Then the function $Y=Y(W)$ is invertible. Indeed, if $\lambda \leqslant 0, Y(W)$ is injective for any $W$, while for $\lambda>0$ it is injective for $W^{2} \leqslant 1 / \lambda \kappa$, that is, $\left(A^{\prime}\right)^{2} \leqslant \beta^{2} / \lambda \kappa$. Thus, in these cases we can view $W$ and $V$ as functions of $Y$.

Note that (28) arises in a non-gravitational theory described by the following action:

$$
\mathcal{S}=\int d^{D} x\left[-\frac{4}{D-2}(\partial \phi)^{2}-\mathcal{V}\right]
$$

where

$$
\mathcal{V} \equiv Y_{\phi}^{2}
$$

Thus, a solution of (28) describes a BPS solution in the theory (30) which depends only on $y$. The tension of the corresponding domain wall is given by

$$
T=\frac{2}{\alpha}[Y(y=+\infty)-Y(y=-\infty)] .
$$

If the theory is supersymmetric, then this (up to a normalization constant) also gives the corresponding central charge, and $Y$ is interpreted as the superpotential.

Next, let us discuss the general condition for such domain walls to be non-singular. That is, we would like to find the condition under which $\phi$ does not blow up at finite values of $y$. First, let as assume that $Y_{\phi}$ does not vanish

\footnotetext{
5 We will refer to the corresponding domain walls as non-singular. However, some of such solutions are actually singular in the sense that the $D$-dimensional Ricci scalar $R$ blows up, but the singularities are located at $y= \pm \infty$ (see below).
} 
for any $\phi$. Then for the domain wall to be non-singular, it is necessary and sufficient that the function

$$
F(\phi) \equiv \int \frac{d \phi}{Y_{\phi}}
$$

is unbounded at $\phi \rightarrow \pm \infty$. That is, the non-singularity condition reads:

- $Y$ should not ${ }^{6}$ grow faster than $\phi^{2}$ for $\phi \rightarrow \pm \infty$, or, equivalently,

- $W$ should not grow faster than $\phi^{2 / 3}$ for $\phi \rightarrow \pm \infty$.

On the other hand, if $Y_{\phi}$ vanishes at one point, ${ }^{7}$ call it $\phi_{0}$, then we have non-singular domain walls interpolating between $\phi=\phi_{0}$ and $\phi= \pm \infty$ as long as at $\phi= \pm \infty$ the above non-singularity condition is satisfied. Finally, if $Y_{\phi}$ vanishes for more then one value of $\phi$, then we have the usual non-singular domain walls of the kink type interpolating between the adjacent values of $\phi$ where $Y_{\phi}$ vanishes. Note that such domain walls have finite tension. In contrast, non-singular solutions where $Y$ goes to $\pm \infty$ have infinite tension. Such domain walls automatically localize gravity (provided that $Y=Y(W)$ is invertible) as long as $W$ changes sign. On the other hand, for the kink type of solutions to localize gravity it is also required that $W$ does not vanish at the edges of the domain wall (that is, at the points where $Y_{\phi}$ vanishes).

\subsection{Positivity conditions}

In this subsection we would like to discuss an additional consistency condition on domain wall solutions in the presence of the Gauss-Bonnet term. Thus, since we are dealing with higher curvature terms, we must make sure that unitarity is not violated in the corresponding warped backgrounds. As in the previous subsection, let us focus on non-singular solutions that localize gravity.

Let us substitute the domain wall ansatz (with $\tilde{\Lambda}=\tilde{\chi}=0$ ) into the action $S$ given by (1). We then obtain the following $(D-1)$-dimensional action for the metric $\tilde{g}_{\mu \nu}\left(x^{\sigma}\right)$ :

$$
\frac{\tilde{S}}{\widetilde{M}_{P}^{D-3}}=\int d^{D-1} x \sqrt{-\tilde{g}}\left[\widetilde{R}+\tilde{\lambda}\left(\widetilde{R}^{2}-4 \widetilde{R}_{\mu \nu}^{2}+\widetilde{R}_{\mu \nu \rho \sigma}^{2}\right)\right],
$$

where we have dropped the boundary terms as they vanish for non-singular domain walls that localize gravity (for such domain walls $A^{\prime} \exp (A)$ and $A^{\prime \prime} \exp (2 A)$ go to zero at $y \rightarrow \pm \infty$ ). In the last equation we are using the following notations:

$$
\begin{aligned}
& \tilde{M}_{P}^{D-3} \equiv M_{P}^{D-2} \int d y \exp [(D-3) A]\left[1+2 \lambda(D-3)(D-4)\left(A^{\prime}\right)^{2}\right], \\
& \tilde{\lambda} \equiv \lambda \frac{M_{P}^{D-2}}{\widetilde{M}_{P}^{D-3}} \int d y \exp [(D-5) A] .
\end{aligned}
$$

The quantity $\widetilde{M}_{P}$ is interpreted as the $(D-1)$-dimensional Planck scale, and $\tilde{\lambda}$ is the $(D-1)$-dimensional analog of $\lambda$. Note that in $D=5$ the quantity $\tilde{\lambda}$ is infinite. This, however, does not pose a problem as in $D-1=4$ dimensions the Gauss-Bonnet term is a total derivative, and if we drop the corresponding topological term, we obtain the usual 4-dimensional Einstein-Hilbert action:

$$
\tilde{S}=\tilde{M}_{P}^{2} \int d^{4} x \sqrt{-\tilde{g}} \widetilde{R}
$$

\footnotetext{
6 More precisely, this is correct up to usual "logarithmic" factors (that is, $\log (\phi), \log (\log (\phi))$, etc., or, more generally, the appropriate products thereof). Thus, for instance, the non-singularity condition on (33) is satisfied for $Y=\xi \phi^{2} \log (\phi)$.

7 Here such a point can be at finite $\phi$ or $\phi= \pm \infty$.
} 
For $D>5$ the Gauss-Bonnet term is no longer a total derivative, and $\tilde{\lambda}$ is finite. Note, however, that if we expand the Gauss-Bonnet term around the flat Minkowski solution (and this is the $(D-1)$-dimensional background we must consider in accord with the original domain wall solution), it does not modify the graviton propagator (that is, the terms quadratic in metric fluctuations arising from expanding the Gauss-Bonnet term combine into a total derivative), so that unitarity is not violated $[17,18]$. Nonetheless, the Gauss-Bonnet term does non-trivially modify the interactions.

The above observation, however, is insufficient to ensure positivity. Thus, the integrand in (36) is positive-definite if $\lambda>0$, but for $\lambda<0$ it can become negative. This implies that $\widetilde{M}_{P}^{D-3}$ can in some cases be negative if $\lambda<0$. We would then have negative-norm states, which violate unitarity. In fact, to ensure unitarity we should require that the integrand in (36) is positive-definite for all $y$, indeed,

$$
\exp [(D-3) A]\left[1+2 \lambda(D-3)(D-4)\left(A^{\prime}\right)^{2}\right]
$$

is interpreted as the square of (the $y$-dependent part of) the graviton wave-function. This then implies the following positivity condition:

$$
\left(A^{\prime}\right)^{2} \leqslant \beta^{2} /|\lambda| \kappa,
$$

or, equivalently,

$$
W^{2} \leqslant 1 /|\lambda| \kappa
$$

Note that for $\lambda>0$ this is a necessary condition for a domain wall to localize gravity. On the other hand, for $\lambda<0$ this condition ensures unitarity.

The above positivity condition has an important implication. Thus, it is not difficult to see that infinite tension domain walls discussed in the previous subsection exist only for $\lambda<0$, and they violate the positivity condition (40). That is, as was already suspected in [16], infinite tension domain wall solutions are not completely consistent once higher curvature terms are included. The reason for this is that such domain walls are actually singular with the singularities (where the Ricci scalar $R$ diverges) located at $y= \pm \infty$. In contrast, flat domain walls with finite tension are non-singular everywhere, and as long as (40) is satisfied (on the solution), they do not violate unitarity. This implies that (for both $\lambda<0$ and $\lambda>0$ ) on the solution we have

$$
V \geqslant-|\eta|,
$$

that is, the scalar potential is bounded below.

\subsection{An example}

For illustrative purposes let us end our discussion here with a simple example of a domain wall with finite tension which satisfies the consistency conditions discussed in this section. Thus, let $\lambda>0$, and let $W=\zeta \phi$ (for definiteness let us assume $\zeta>0$ ). We then have

$$
Y=\zeta \phi-\frac{\lambda \kappa}{3} \zeta^{3} \phi^{3}
$$

The domain wall solution is then given by:

$$
\begin{aligned}
& \phi(y)=\frac{1}{\zeta \sqrt{\lambda \kappa}} \tanh \left[\alpha \zeta^{2} \sqrt{\lambda \kappa}\left(y-y_{0}\right)\right], \\
& A(y)=\frac{\beta}{\alpha \zeta^{2} \lambda \kappa} \ln \left(\cosh \left[\alpha \zeta^{2} \sqrt{\lambda \kappa}\left(y-y_{0}\right)\right]\right)+A_{0},
\end{aligned}
$$

where $y_{0}$ and $A_{0}$ are integration constants. 


\section{Comments}

In this section we would like to make a few concluding remarks. As we saw in the previous section, consistent flat domain wall solutions in the presence of the Gauss-Bonnet term are of the kink type, and they interpolate between adjacent AdS minima of the scalar potential. Here we should point out that such solutions always have consistent curved deformations (that is, for such potentials there always exist consistent domain wall solutions with non-vanishing $(D-1)$-dimensional cosmological constant).

As we have already mentioned in introduction, one of the motivations for choosing the Gauss-Bonnet combination is that, as was pointed out in [16], generic higher curvature terms actually delocalize gravity. Thus, inclusion of higher derivative terms of, say, the form

$$
\zeta \int d^{D} x \sqrt{-G} R^{k}
$$

into the bulk action would produce terms of the form [16]

$$
\zeta \int d^{D-1} x d y \exp [(D-2 k-1) A] \sqrt{-\tilde{g}} \widetilde{R}^{k} .
$$

Assuming that $A$ goes to $-\infty$ at $y \rightarrow \pm \infty$, for large enough $k$ the factor $\exp [(D-2 k-1) A]$ diverges, so that at the end of the day gravity is no longer localized. In fact, for $D=5$ delocalization of gravity takes place already at the four-derivative level once we include the $R^{2}, R_{M N}^{2}$ and $R_{M N R S}^{2}$ terms with generic coefficients (with the only exception being the Gauss-Bonnet combination).

A possible way around this difficulty might be that all the higher curvature terms should come in "topological" combinations (corresponding to Euler invariants such as the Gauss-Bonnet term [17,18]) so that their presence does not modify the $(D-1)$-dimensional propagator for the bulk graviton modes. That is, even though such terms are multiplied by diverging powers of the warp factor, they are still harmless. One could attempt to justify the fact that higher curvature bulk terms must arise only in such combinations by the fact that otherwise the bulk theory would be inconsistent to begin with due to the presence of ghosts. However, it is not completely obvious whether it is necessary to have only such combinations to preserve unitarity. Thus, in a non-local theory such as string theory unitarity might be preserved, even though at each higher derivative order there are non-unitary terms, due to a non-trivial cancellation between an infinite tower of such terms.

We would like to end our discussion by pointing out that the aforementioned difficulty with higher curvature terms does not arise in theories with infinite-volume non-compact extra dimensions [24-30]. However, in such scenarios consistency of the coupling between bulk gravity and brane matter might give rise to additional constraints. Thus, in some cases the brane world-volume theory must be conformal [29]. In such cases it would be interesting to understand if there is a relation to [31].

\section{Acknowledgement}

This work was supported in part by the National Science Foundation. Z.K. would like to thank Albert and Ribena Yu for financial support.

\section{References}

[1] V. Rubakov, M. Shaposhnikov, Phys. Lett. B 125 (1983) 136.

[2] A. Barnaveli, O. Kancheli, Sov. J. Nucl. Phys. 52 (1990) 576.

[3] J. Polchinski, Phys. Rev. Lett. 75 (1995) 4724. 
[4] P. Hořava, E. Witten, Nucl. Phys. B 460 (1996) 506;

P. Hor̆ava, E. Witten, Nucl. Phys. B 475 (1996) 94;

E. Witten, Nucl. Phys. B 471 (1996) 135.

[5] J. Lykken, Phys. Rev. D 54 (1996) 3693.

[6] G. Dvali, M. Shifman, Nucl. Phys. B 504 (1997) 127;

G. Dvali, M. Shifman, Phys. Lett. B 396 (1997) 64.

[7] N. Arkani-Hamed, S. Dimopoulos, G. Dvali, Phys. Lett. B 429 (1998) 263; N. Arkani-Hamed, S. Dimopoulos, G. Dvali, Phys. Rev. D 59 (1999) 086004.

[8] K.R. Dienes, E. Dudas, T. Gherghetta, Phys. Lett. B 436 (1998) 55;

K.R. Dienes, E. Dudas, T. Gherghetta, Nucl. Phys. B 537 (1999) 47, hep-ph/9807522;

Z. Kakushadze, Nucl. Phys. B 548 (1999) 205;

Z. Kakushadze, Nucl. Phys. B 552 (1999) 3;

Z. Kakushadze, T.R. Taylor, Nucl. Phys. B 562 (1999) 78.

[9] Z. Kakushadze, Phys. Lett. B 434 (1998) 269;

Z. Kakushadze, Nucl. Phys. B 535 (1998) 311;

Z. Kakushadze, Phys. Rev. D 58 (1998) 101901

[10] I. Antoniadis, N. Arkani-Hamed, S. Dimopoulos, G. Dvali, Phys. Lett. B 436 (1998) 257.

[11] G. Shiu, S.-H.H. Tye, Phys. Rev. D 58 (1998) 106007.

[12] Z. Kakushadze, S.-H.H. Tye, Nucl. Phys. B 548 (1999) 180;

Z. Kakushadze, S.-H.H. Tye, Phys. Rev. D 58 (1998) 126001.

[13] M. Gogberashvili, hep-ph/9812296;

M. Gogberashvili, Europhys. Lett. 49 (2000) 396.

[14] M. Visser, Phys. Lett. B 159 (1985) 22;

P. van Nieuwenhuizen, N.P. Warner, Commun. Math. Phys. 99 (1985) 141.

[15] L. Randall, R. Sundrum, Phys. Rev. Lett. 83 (1999) 3370;

L. Randall, R. Sundrum, Phys. Rev. Lett. 83 (1999) 4690.

[16] Z. Kakushadze, Nucl. Phys. B (in press), hep-th/0005217.

[17] B. Zwiebach, Phys. Lett. B 156 (1985) 315.

[18] B. Zumino, Phys. Rep. 137 (1986) 109.

[19] I. Low, A. Zee, hep-th/0004124.

[20] N.E. Mavromatos, J. Rizos, hep-th/0008074.

[21] I.P. Neupane, hep-th/0008190.

[22] Z. Kakushadze, hep-th/0008128.

[23] D.Z. Freedman, S.S. Gubser, K. Pilch, N.P. Warner, JHEP 0007 (2000) 038.

[24] R. Gregory, V.A. Rubakov, S.M. Sibiryakov, Phys. Rev. Lett. 84 (2000) 5928.

[25] C. Csaki, J. Erlich, T.J. Hollowood, Phys. Rev. Lett. 84 (2000) 5932.

[26] G. Dvali, G. Gabadadze, M. Porrati, Phys. Lett. B 484 (2000) 112;

G. Dvali, G. Gabadadze, M. Porrati, Phys. Lett. B 484 (2000) 129;

G. Dvali, G. Gabadadze, M. Porrati, Phys. Lett. B 485 (2000) 208;

G. Dvali, G. Gabadadze, M. Porrati, hep-ph/0007211.

[27] E. Witten, hep-ph/0002297.

[28] G. Dvali, hep-th/0004057.

[29] Z. Kakushadze, hep-th/0006059;

Z. Kakushadze, hep-th/0006215;

Z. Kakushadze, hep-th/0008041.

[30] G. Dvali, G. Gabadadze, hep-th/0008054.

[31] M. Bershadsky, Z. Kakushadze, C. Vafa, Nucl. Phys. B 523 (1998) 59;

Z. Kakushadze, Nucl. Phys. B 529 (1998) 157;

Z. Kakushadze, Phys. Rev. D 58 (1998) 106003;

Z. Kakushadze, Phys. Rev. D 59 (1999) 045007;

Z. Kakushadze, Nucl. Phys. B 544 (1999) 265. 